\title{
Seismic Data Qualifying for Fracture Detection Along E\&P Interpretation Processes
}

Rogério Santos - UFF, Jose Ricardo Bastos - Schlumberger, Eliane Alves - UFF and Marcelo Alvarenga- Schlumberger

\section{Copyright 2019, SBGf - Sociedade Brasileira de Geofísica}

This paper was prepared for presentation during the $16^{\text {th }}$ International Congress of the Brazilian Geophysical Society held in Rio de Janeiro, Brazil, 19-22 August 2019.

Contents of this paper were reviewed by the Technical Committee of the $16^{\text {th }}$ International Congress of the Brazilian Geophysical Society and do not necessarily represent any position of the SBGf, its officers or members. Electronic reproduction or storage of any part of this paper for commercial purposes without the written consent of the Brazilian Geophysical Society is prohibited.

\section{Abstract}

The importance of seismic analysis of natural fractures in hydrocarbon reservoirs has grown in line with the increasing economic demands of the $E$ \& $P$ processes. Such importance is associated with geomechanical, faciological, permeability, porosity, seal efficiency, reservoir construction and hydrocarbon migration routes, among others. In the initial stages of exploration, the correlation of the existence of natural fractures and their properties have huge importance, with zones of fractures, with structural deformations in response to regional tectonic efforts, world stress, when these fractures sometimes need to be defined counting only with 2D seismic data, with low spatial resolution, or geological, numerical and surface models. In processes of development of production, natural fractures gain importance for the geomechanical definition of the petroleum system involved, permeability capacities, and reservoir saturation, whether in the conventional or unconventional form. For the latter, natural fractures gain importance because they have a critical role in the performance and economicity of the development plan, because based on it are applied methods of stimulation for its effective production. In the exploratory phase, fracture interpretation methods in seismic data generally use generic mathematical algorithms to detect structural heterogeneities such as numerical correlation, vector similarity, variance, neighboring sample set curvatures, and others. However, the resolution needed to succeed in obtaining seismic patterns correlated with events associated with natural fractures needs to be much greater than when they are applied, for example, to faults. In addition, the geological knowledge of the interpreter in seismic patterns that are correlated to possible geological features is fundamental in the choice and construction of each method or algorithm applied. In this phase, the interpretation of fractures zones of regional distribution and the efficiency of seals are gaining importance. When we enter the production development phase, where a macro configuration of the spatial distribution of the reservoirs is already available, correlations of lithological, petrophysical and fracture parameters observed in wells with elastic and acoustic properties generally observed in seismic are sought. Major advances in the identification of fracture zones have been achieved in the search for answers that define important factors related to structural discontinuities and geomechanical behavior of reservoirs. However, one of the great limitations for any algorithm applied for fracture detection from seismic data is related to two critical technical factors: an intrinsic restriction related to the seismic data resolution and the amount of undesirable coherent events involved. In this work, we propose technical aspects related to the benefits that can be achieved when seismic patterns for fracture detection algorithms, even those conventional, are analysed after a previous data qualifying. Results are exemplified in seismicdata from Teapot Dome field (USA), pointing out differences in the performance of detection algorithms under different amplitude levels of undesirable events, coherent and uncoherent.

\section{Introduction}

Fracture analysis from seismic data can be logically grouped similarly to the forms that are divided the seismic interpretation stages within the chain of E\&P processes: In stages of exploration, fracture interpretation methods are focused on the detection of structural heterogeneities that impact on the construction and effectiveness of the involved oil system. Events such as natural fractures can occur within conventional reservoir rocks acting as intrinsic porosity and permeability factors during their 
production. But natural fractures can occur also within low permeability rocks, being source and reservoir simultaneously, in unconventional way, interfering in permeability and expulsion of fluids from such rocks. Besides, fractures have important role on the understanding of hydrocarbon migration routes, especially those associated with vertical fractures. They still have crucial importance in seal efficiency. The required seismic visual resolution to succeed the definition of seismic patterns effectively correlated with natural fractures, is generally much higher than that demanded for fault detection. Due to this aspect, modern artificial intelligence algorithms such as machine learning, and purely automatic methods for structure detection, sometimes have not been successful in detecting fractures, needing geological support, interactive and iterative, from interpretera regarding the definition of seismic patterns correlated to geological or petrophysics patterns.

During the exploitation phases, there is usually a control of spatial distribution of reservoirs, with their seismic distributions associated with possible production zones, correlated to stratigraphic zones, and for which realistic distributions of lithological, petrophysical and fracture properties are sought, gradually adjusted by the samples of drilled wells. These distributions are desirably associated with elastic and acoustic properties observed in the seismic data through the time of production (4D seismic). Also in the exploitation phase, resolution limits of seismic data are obstacles to identify natural fractures, even when data is acquired with denser sampling and with increasingly modern acquisition techniques. Even so, good results have been described along specialized bibliography to define possible zones of natural fractures, which would be indicated by attributes that point out the best facies in which fractures may occur, even if it is not possible to indicate with high precision if a region would be, or not, already fractured. However, one strong performance constrain for all algorithms applied in the definition of seismic fractures is related to two critical technical factors: intrinsic limits of resolution and amount of coherent noise in each data. In this work, we describe technical aspects related to the benefits achieved when algorithms for seismic fracture detection act are applied over data which had been previously qualified for such detection, atenuating coherent and uncoherent noises and also undesirable signals. The objective is defining parameters for seismic qualifying and show that it is always possible to construct methods of treatment and analysis of seismic data to obtain increasingly accurate responses: the here called qualifying levels. ven in mature fields or fields that are entering production, which have already been heavily exploited in terms of the best seismic technologies applied, there is always room for new experiments that can bring substantial economic benefits. Seismic qualifying results are shown on data from the Teapot Dome, presenting differentiations in the performance of detection applications for different levels of coherent noise attenuation. incoherent noises and undesirable signals. In such a basin, the Tensleep Formation has been exhaustively analyzed using seismic data to evaluate factors related to fracture detection, and one question that may arise is: would still be possible to investigate or learn something more from such seismic data?

\section{Theory for Seismic Disorder}

Many seismic methods for detecting structural heterogeneities have been developed over the last decades, most of them dedicated to the definition of faults. When we want to analyze structural heterogeneities, another way of thinking the 3D seismic data being composed by textural features. An important work was presented by Signer et al (1998) for fracture characterization at the reservoir scale combined with seismic facies mapping. They mention that, already in that epoch, interpretations of fault systems from 3D seismic data is highly time consuming and that vertical and horizontal extent of the faults are many times difficult to interpret, especially in complex faulted area like in carbonate fractured reservoirs. They proposed an approach that overcome these challenges to extract automatically and precisely fault network from reservoir layers. An important attribute in this sense was derived for the so-called chaos presented by Randen et al. (2001), based on eigen-analysis of gradient covariance. Being a seismic disorder attribute it is based on application of second derivative operators in the three axial directions in a 3D seismic volume. Mathematically, it is related to eigenanalysis of the $3 \times 3$ gradient structure tensor. When the first eigenvalue is large, the corresponding first eigenvector defines the normal to a local plane of constant amplitude waveforms. When the three eigenvalues are equal, the data are totally chaotic. Intermediate values of chaos indicate the degree of data organization. To be successful in detecting fracture zones, it is important to qualify the geology, geophysics, and data that will be interpreted within zones of amplitude disorder. In the case of fracture detection, some amplitude questions should 
be evaluated: What type of tectonic effort is expected in the study region? What is the level of amplitudes found in generic structural heterogeneities? What is the relative level of amplitudes that distinguishes fractures from faults? What are the noises present in the seismic data that contaminate the primary seismic response and reduce the performance of a certain algorithm? What level of resolution does it take to succeed in defining a vertical event continuity? The algorithms applied in the detection presuppose the existence of wavelets in the seismic trace? All these questions go through the analysis not only of incoherent and coherent noises that are present in a given seismic data, but also of undesirable signals. In this sense, the concept of qualifying of the seismic data is here established.

\section{Qualifying the Seismic Data}

A seismic data has the exact value that each interpreter can assign to it, depending on the quantity and quality of the information extracted from it. For different interpreters, the same seismic data has completely different values. When it is desired to qualify a given seismic data, in terms of its possibilities in which it can effectively respond to the demands of a particular interpretative task, it becomes necessary to analyze minimally: the geology involved, the environment of the acquisition, the depths of the geological targets, the operational quality of the seismic data to be interpreted (for both in the acquisition and in the seismic processing) and the interpretative qualities of the analyzed seismic data. In a seismic data qualifying, one must focus on the target that wants to interpret. Here in this work, as an example of this simple observation, our targets are "seismic fractures at great geological depths". After this choice, we should qualify our data only for this unique conceptual target.

\section{Seismic data qualifying is not Seismic Data Conditioning}

While seismic data conditioning flows generally propose filters, residual static and other processes, in order to attenuate generic noise and enhance all signals, data qualifying proposes to attenuate specific seismic effects on the data, which may be a noise or even an undesirable signal. In this way, we must qualify the data and attenuate those amplitudes for all the events that contaminate the chosen event we wish to preserve. Generically, this process aims to improve the quality of facies and seismic textures. This task would be more difficult if the data is not filtered for qualified amplitudes for such improvement.
A geoscientific interpretation that includes data qualifying expands the chance of success of seismic detection for heterogeneities related to, for example, stratigraphic and petrophysical features.

\section{Method for Seismic Qualifying}

Considering the interpretation of two adjacent traces, the process of data qualifying for heterogeneity detection, or for any other objective, should analyze both traces having as reference a global relative amplitude value, derived from a common input signal $\mathrm{s}(\mathrm{t})$, and different additive noises $\mathrm{n}(\mathrm{t})$ and $\mathrm{m}(\mathrm{t})$ :

$x(t)=s_{x}(t)+n(t)$

and

$y(t)=s_{y}(t)+m(t)$

where $s_{x}(t)$ and $s_{y}(t)$ are the seismic responses from a geological target, in samples $[(x, y), t]$, to a wave propagation related to signal $\mathrm{s}(\mathrm{t})$. The analysis of noises, present in both traces, can be summarized in a differentiation between $n(t)$ and $m(t)$, treated as additive incoherent noises and which are generally very well attenuated in most current seismic processes. On the other hand, if we consider noises wich are convolved with $s(t)$ along propagation, having different spectra, $c_{\mathbf{x}}(\mathrm{t})$ and $c_{\mathbf{y}}(\mathrm{t})$, the seismic traces in equations (1) and (2), will have the following form:

$x(t)=\left(s_{x}(t)^{*} C_{x}(t)\right)+n(t)$

and

$y(t)=\left(s_{y}(t)^{*} c_{y}(t)\right)+m(t)$

where $c_{x}(t)$ e $c_{y}(t)$ are considered coherent noises normally associated to multiples (long and short periods), wavelet effects, mode conversion, and effects from surveys and processing. For many data, these coherent noises are not satisfactorily attenuated by filters like a global function $F(t)$ and are always present in several interpreted seismic volumes, even for those demanding higher seismic resolution. Such coherent noises are more signal destructive for many geological targets when the energy level of the input signal $s(t)$ is considered relatively low and/or when the reflectivities of targets are small. We can extend the qualifying seismic data process, including undesirable signals. Then $F(t)$ is the qualifying operator that will help the efficiency of any algorithm for fracture detection. With this process, there is a controlled smoothing of the amplitudes, reducing the deleterious effects of high amplitude, all considered noise, and 
improving the image of some geologically critically associated to the regions with stronger amplitudes. A very efficient way to evaluate the performance of spatial filters is to calculate the chaos between seismic traces, before and after specific process. In practice, disorder can be used to isolate discontinuities evaluated for lateral continuity of amplitudes. This is also suitable for petrophysical, stratigraphic and structural attributes and can bring out aspects about facies and depositional features, including terrigenous, carbonates, evaporites and igneous rocks, besides faults and fracture zones. In the case here studied, the chaos is adopted to observe the gain of resolution after $F(t)$ filtering processes, perceived by the focusing and by temporal and lateral consistency of textures. These aspects are exemplified for Teapot Dome area.

\section{Geologic Setting for Teapot Dome}

Teapot Dome is located in central Wyoming and its structure is a doubly plunging basement-cored anticline of Laramide-age (Fox et al., 1991) being a part of a larger anticlinal system that includes the Salt Creek anticline to the north. Oil was first produced in 1908. Historically, oil has been produced from 9 different reservoirs. Here is analyzed the deepest, Pennsylvanian age Tensleep Formation, our targetreservoir. The combined cumulative oil production from this anticlinal system was $675 \mathrm{MMBO}$ in 1990. Such production, using only vertical wells, were influenced by the presence of natural fractures.

\section{Complex fracture development in Tensleep Eolian Sandstone}

In the study area and for exploration phase, Zahm and Hennings (2009) show that methods of predicting fractures commonly neglect to include the stratigraphic architecture as part of the prediction or characterization process. This may be critical as complex heterogeneous fracture development occurs within the eolian Tensleep Sandstone. According to the authors, the heterogeneous fracture development occurs at four scales of observation: Lamina-bound; facies-bound; sequence-bound and throughgoing fractures that span the formation. Ghahfarokhi e Wilson (2015) mention that in TeaPot Dome qualitative correlation of production data to extracted discontinuities suggests that wells located on hingeoblique discontinuities are more productive than other wells in the field. They built a workflow for fracture detection including the most negative curvature attribute that highlights concave features attributed to subtle travel time delay through fracture zones and small faults or flexures associated with the fracture swarms. Their post stack discontinuity extraction workflow also incorporated seismic spectral blueing to enhance the resolution of the seismic data, followed by computation of the short-wavelength most negative curvature. Subsequently, the minimum similarity attribute is applied to accentuate regions with minimum similarity of curvature. An edge-illumination process was then applied to the minimum similarity of the most negative curvature output. Discontinuities extracted through edge illumination locate regions of minimal similarity in curvature along fracture zones or small fault boundaries. According to the authors, this extensive workflow enhances hinge-oblique discontinuities without azimuthal filtering and provides a fracture intensity attribute, which is used as an input to distribute the fracture intensity through the model discrete fracture network.

For the exploitation phase, Ouenes et al (2010) describes a workflow that fully utilizes the post-stack seismic attributes to derive reliable geologic and fracture models that are validated with multiple blind wells and reservoir simulation. The first step in the workflow is to run post-stack seismic processes, which includes volumetric curvature, post-stack inversion and spectral imaging. The second step consists of using the various post-stack seismic attributes to derive 3D geologic and fracture models. The third step is to use the derived models in a reservoir simulator to verify the validity of the models. This workflow was applied to the Tensleep Formation. An effective permeability was estimated by using a linear combination of the scaled fracture density and the matrix permeability.

A basic question for both phases, reservoir exploration and exploitation, can be defined from the point of view of the extreme complexity of workflows in order to obtain a definition of low amplitude heterogeneities and highly influenced by data disorder. The idea here presented for seismic data qualifying is to simplify the processes, let the interpreter act, focusing uniquely on the desired signal and attenuate all the rest of the events, being it noise or undesirable signal.

\section{Examples and Results}

For an example of qualifying $F(t)$ aplication, we have used poststack seismic attributes to describe the fracture network of the naturally fractured Tensleep Formation. The attributes include chaos and heterogeneities detection processes, like ants and coherence algorithms 
We present qualifying results after processes applied to original post stack data, exemplified here in a TWT slice in figure 01

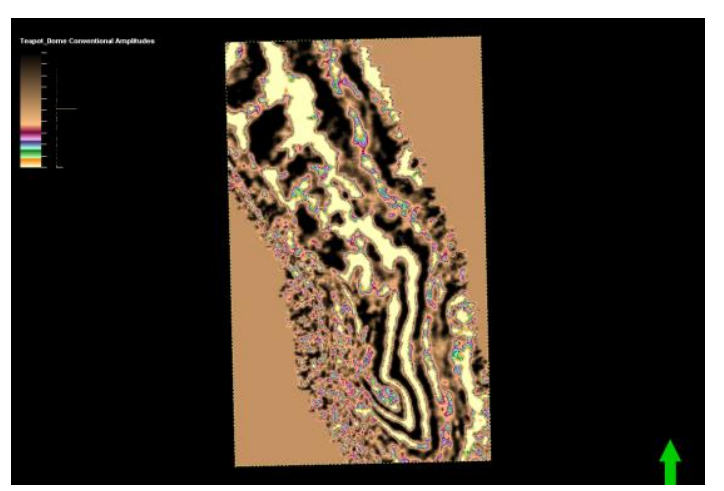

Figure 01 - TWT (ms) slice for amplitudes nearby the top of Tensleep Formation

To identify the performance of seismic data qualifying applied over original data, we use the disorder approach described by chaos process. Figure 02 illustrate such results related to the data as shown in figure 01.

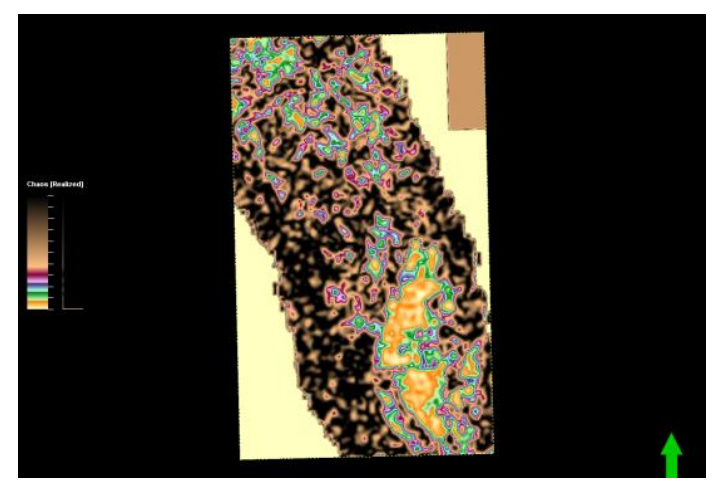

Figure 02 - TWT (ms) slice for chaos applied to the amplitudes nearby the top of Tensleep Formation, as shown in figure 01

Finally, exemplifying some fault and fractures detected from disorder information as shown in figure 02, we applied ants processes to have an approach for modeling faults and fractures from a such data, like shown in figure 03.

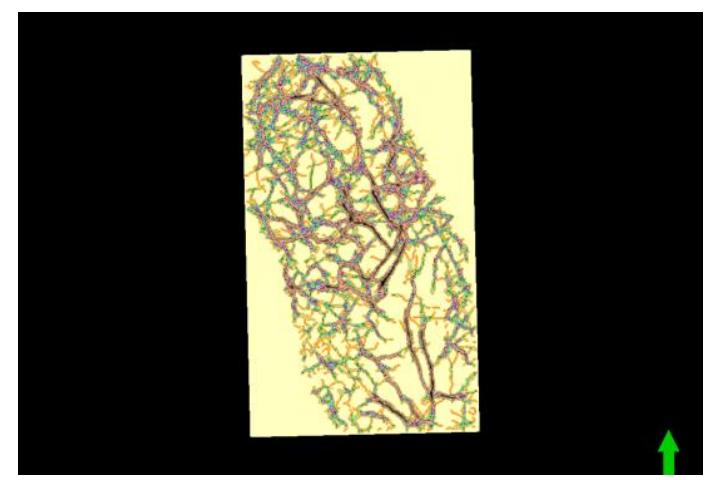

Figure 03 - TWT (ms) slice after ants process applied over chaos results nearby the top of Tensleep Formation, as shown in figure 02.

In this work we show how these processes can be improved after qualifying the seismic data.

\section{Conclusions}

Seismic qualifying for frature detection, as presented here, is the process that focuses only on the desirable amplitudes for a given signal. For this process, all undesirable seismic events, whether they are noises or signals, are attenuated. The results obtained applying this process have shown excellent efficacy in determining more precisely low-amplitude, structural or petrophysical heterogeneities with low energy levels and for which the seismic samples disorder does not allow good performance for many applications that seek to identify them using conventional data but without such qualifying.

\section{Acknowledgments}

The authors express many thanks to The U.S. Department of Energy that had made its RMOTC data sets available for use in scientific research, Schlumberger for permission to publish these results.

\section{References}

FOX, J.E., DOLTON, G.L.. and CLAYTON, J.L. Powder River Basin, in Gluskoter, Rice, H.J.,D.D. and Taylor, R.B.. eds. Economic Geology, U.S.: Geolocial Society of America, The Geology of North America, P2, p.373-390.1991, 
OUENES, A., ANDERSON,T., KLEPACKI, D., BACHIR, A., BOUKHELF, A., ARAKTINGI, U., HOLMES, M., BLACK, B., and STAMP.V. Integrated Characterization and Simulation of the Fractured Tensleep Reservoir at Teapot Dome for CO2 Injection Design, SPE132404, 2010.

SIGNER, C., NICKEL, M., RANDEN,T., OSTEBO, M., SONNELAND, L.; REYMON,B.; SAETER, T.; and VEIRE, H.H.. Automated seismic fracture extraction and analysis for reservoir characterization. SEG Expanded Abstracts, 1998.

RANDEN, T., PEDERSEN, S. I., and SONNELAND, L., Automatic Extraction of Fault Surfaces from ThreeDimensional Seismic Data, Ann. Int. Mtg., Soc. Expl. Geophys., Exp. Abstr, 2001.

ZAHM, C.K. and HENNINGS, P.H.. 3D seismic curvature and flexure for unconventional fractured reservoir characterization at Teapot Dome (Wyoming), SEG 2009 